\title{
Wie kann der Staat Vertrauen organisieren?
}

Moritz Leuenberger

\section{Vertrauen und Misstrauen}

Ohne Vertrauen in andere können wir uns nicht entfalten und deshalb nicht in Freiheit leben. Und es gilt umgekehrt: Ohne Misstrauen können wir uns nicht entfalten, wir würden unweigerlich zum Opfer auf der Wildbahn von Ausbeutern. Deswegen misstraut der wachsame Mensch seinem Gegenüber. Wir sprechen daher von "gesundem« Misstrauen. Um selber zu überleben und um miteinander zu leben, brauchen wir beides, Vertrauen und Misstrauen.

OhneVertrauen keine Freiheit. Ohne Misstrauen keine Sicherheit. Willhelm Busch umschrieb das so:

»Wer andern gar zu wenig traut, hat Angst an allen Ecken;

Wer gar zu viel auf andre baut, erwacht mit Schrecken«

Was zwischen mir und dir gilt, gilt für alle, für die Organisation einer demokratischen Gemeinschaft. Sie kann nur funktionieren, wenn sie auf dem Grundvertrauen zwischen den Menschen aufbaut und dieses fördert und sichert.

Aber sie darf dabei nicht von einem idealistischen Menschenbild ausgehen, sondern muss in Rechenschaft ziehen, dass es Menschen gibt, die Vertrauen missbrauchen.

\section{Das Gesetz verlangt Vertrauen}

Die schweizerische Gesetzgebung versucht, Treu und Glauben als Grundlage zwischen den Menschen umfassend zu etablieren. Sie hütet sich dabei, einzig und allen auf das Gute in den Menschen $\mathrm{zu}$ vertrauen, sondern sie geht davon aus, dass das Vertrauen missbraucht wird. Beinahe durchwegs wird dabei das folgende Prinzip angewendet:

Wir gehen davon aus, im Allgemeinen sei bei der grossen Mehrheit der Bürger ein gegenseitiges Grundvertrauen verwurzelt - aufgrund ihrer Erziehung, ihrer Kultur und Religionen. Dies kommt gesetzgeberisch in der sogenannten Freiwilligkeit zum Ausdruck. 
Das Gesetz sieht, um dieses Grundvertrauen zu stärken und zu verfestigen, Anreize und Sanktionen vor, um vertrauensvolles Verhalten zu fördern (Ungültigkeit von Verträgen, Schadenersatz etc)

Und es sieht Zwangsmassnahmen bis und mit strafrechtlichen Sanktionen vor.

Dieses Prinzip findet sich im Zivilrecht, im Bankenwesen, im Umweltrecht.

Im Zivilrecht wird die eheliche Untreue verboten. In Handel, Wirtschaft und Vertragswesen wird der gute Glaube geschützt mit Schadenersatzforderungen oder Ungültigkeit der Verträge als Folge. Um als Bergfuihrer und Lokomotivführer zu wirken, müssen Ausbildungen absolviert und Vorschriften eingehalten werden, damit nicht Haltesignale überfahren werden wie anfangs Woche in Neuhausen.

(Ihre Tagung läuft unter dem Titel "Zwischen Risiko und Sicherheit - welches Vertrauen brauchen wir?« Einem Flugkapitän und einem Fluglotsen wollen wir absolut vertrauen, dass er die Regeln gewissenhaft einhält. Der Staat erlässt deshalb entsprechend absolute Vorschriften. Dennoch bleibt der menschliche Faktor als grösstes Risiko, wenn wir uns einer Technologie anvertrauen müssen. Bei den Technologien selber sind dagegen Massnahmen möglich, welche jedes Risiko ausschliessen: Gestern wurde ein weltweites Verbot von Dreamlinern erlassen.)

Im ausservertraglichen Bereich wird die Schadenersatzpflicht für unrechtmässige Schädigung geregelt. Im Strassenverkehr gilt ebenfalls das Vertrauensprinzip. Wenn Anzeichen dafür da sind, dass sich ein anderer Teilnehmer vorschriftswidrig verhält, muss darauf Rücksicht genommen werden. (Wenn ein Fussgänger bei rot über die Strasse laufen will, darf der Automobilist nicht auf seinem Vortritt beharren und hupen, sondern er muss bremsen. Der Fussgänger muss darauf vertrauen können, dass man sich auf seinen Fehler einrichtet.)

Treu und Glauben gelten auch im öffentlichen Recht, also im Verhältnis zwischen Staat und Bürger. In die Beamten und Auskunftspersonen soll man sich verlassen dürfen: Ein klassisches Beispiel liefert der Fall Polanski: Es darf nicht ein und derselbe Staat einem Bürger (auch einem Ausländer nicht), gegen den von allem Anfang an ein Auslieferungsgesuch vorliegt, durch den einen Kanton die Bewilligung für einen Hauskauf erteilen, Motorfahrzeugsteuern erheben lassen, ihn während Jahren ein- und ausreisen lassen, ihn dann für die Teilnahme an ein Filmfestival mit Preisübergabe, an dem ein Bundesamt beteiligt ist, einladen, und ihn dann unvermittelt zu verhaften und ihn an die USA ausliefern. Polanski wurde in seinem Vertrauen in die Schweiz vom Bundesrat geschützt. 


\section{Das Vertrauen in den Staat und in seine Behörden}

In der Politik wird ganz besonders um Vertrauen geworben, vor allem in das Vertrauen in Personen. Doch das Misstrauen in der Politik ist allgegenwärtig und offensichtlich. In der direkten Demokratie behält sich der Stimmbürger das letzte Wort über Verfassung und Gesetze vor. Das führt denn auch zum beruhigenden Umstand, dass die Wahl in die Regierung nicht derart wichtig ist, weil einzelne Gesetzesvorlagen an der Urne ja verhindert werden können.

Dennoch lebt auch die direkte Demokratie von Repräsentation, vom Vertrauen in Regierungsmitglieder, in Parlamentarier, in Parteipräsidenten und in Parteiparolen. Ohne Vertrauen ist Repräsentativität nicht möglich.

(Ich lege bei Wahlen von Schulpflegern die Liste unverändert ein, weil ich Vertrauen in die interparteiliche Einigung habe.)

Enttäuschungen über das Verhalten gewählter Regierungsmitglieder sind denn in repräsentativen Demokratien auch regelmässig um einiges grösser. Es ist schon beinahe ein politisches Ritual, wie rasch ein siegreicher Kandidat das vorherige Vertrauen, bezw. den Vertrauensvorschuss verliert und in Ungnaden der Umfragen fällt.

Diese Regelmässigkeit führt ganz allgemein zu einem Schwinden des Vertrauens in die Politik im Allgemeinen und sie führt zu einer eigentlichen Spirale im Ringen um Vertrauen, zu populistischen Versprechen, die nicht zu erfüllen sind - und zu den Enttäuschungen beim Wähler danach.

Das ist je nach organisatorischer Ausgestaltung einer Demokratie beinahe die Regel. Weil zu viel versprochen wird, weil Realitäten ausgeblendet werden, weil zu viel erwartet wird, weil Irreales projiziert wird.

Daher geht die Organisation eines demokratischen Staates von einem Misstrauen aus, es könne die anvertraute Macht missbraucht werden:

Es wird die Gefahr von Machtballung gebrochen durch Gewaltenteilung, durch check and balances oder durch die Rotation des Bundespräsidiums. Das stärkt das Vertrauen in das politische System. Dazu gehört auch, dass Bundesrichter während der Amtszeit nicht abberufen werden können, aber auch Bundesräte nicht. Bei uns gibt es die Vertrauensfrage nicht, weil das Vertrauen in den gewählten Bundesrat von der Verfassung für vier Jahre vorausgesetzt wird. Das ist nichts anderes als ein Vertrauen in das politische System und bedeutet einen Schutz der demokratischen Institution (nicht etwa der Gewählten). Damit soll eine Abwahlmöglichkeit nicht zur Disziplinierung und Medienhatz verkommen und damit im Gegenzug 
die Verantwortung bei der Vertrauensprüfung, nämlich der Wahl, geschärft werde (damit zum Beispiel nicht ein Kandidat auf den Schild gehoben wird, der früher Veruntreuungen begangen hat).

Je besser die Staatsform, desto weniger muss das Vertrauen in die Person strapaziert werden; Da braucht es dann gar nicht mehr besonders gute Politiker, schon gar kein einen dauernden Bundespräsidenten - schon zwei Jahre wären viel zu lang.

Das Vertrauen in politische Systeme und Ideologien einerseits und dasjenige in Personen andererseits stehen in einem wechselseitigen Verhältnis. Das zeigt sich schon im privaten Bereich: Junge Paare wählen das Konkubinat auch deswegen, weil sie die Bindung, das gegenseitige Vertrauen, eben gerade nicht gesetzlich vorgeschrieben, sondern freiwillig aus dem eigenen Herzen erbringen wollen.

Je grösser Vertrauen in Personen, desto kleiner Bewusstsein für Forderungen wie Gewaltenteilung: Kleine Kantone haben zum Teil bis heute Mühe damit. Umgekehrt: Je anonymer und unübersichtlicher eine Gesellschaft, desto ausgeklügelter die Sicherheitsmechanismen gegen Vertrauensmissbrauch.

Und: Populisten, die persönliche Machtfülle und damit grenzenloses Vertrauen für sich verlangen, neigen dazu, das Misstrauen gegen »das System« zu schüren. Je nach politischer Optik wird dieses System dann als globale Kapitalismusmafia oder als classe politique bezeichnet.

Umgekehrt: Je grösser der Vertrauensverlust in einzelne Vertreter wird, desto rascher werden systemische Massnahmen getroffen. Das sehen wir auch in kleineren alltäglichen Vorfällen. Die Reaktion auf den Fehler des Lokführers von Neuhausen ist klassisch: Es sollen neue technische Systeme menschliche Fehler auskorrigieren.

Mein Eintritt in einen Verwaltungsrat einer Baufirma nach dem Rücktritt aus dem Bundesrat hat viele enttäuscht, weil das bisher Bürgerlichen vorbehalten blieb (oder Sozialdemokraten in Deutschland). Deswegen hat heute die vorberatende Kommission des $\mathrm{Na}-$ tionalrates eine zweijährige Karenzfrist entschieden. Ich habe mich umgehende bei Opra Winfrey zwecks Beichte angemeldet und finde überdies die beschlossene Karenzfrist gar nicht schlecht, weil ich unterdessen gemerkt habe, dass es mindestens zwei Jahre braucht, bis man nach dem Rücktritt aus dem Bundesratsleben weiss, was man eigentlich will und soll.

Aus solchen Ereignissen und Entwicklungen zeigen, dass sich das Vertrauen von Personen auf systemische Massnahmen, auf politische Modelle oder auch Ideologien verlagert. 


\section{List und Vertrauen}

Die politischen und moralischen Fragen zeigen sich in der Frage:

Welches Vertrauen wird geschützt oder vorgeschrieben? Denn es herrscht das Gebot auf bedingungsloses Vertrauen so wenig wie es eine absolute Wahrheitspflicht gibt. Es gibt die listige Taktik, die raffinierte Verführung.

Das gilt zwischen den Menschen, das gilt im Wettbewerb, also in Handel und Wirtschaft. Es gibt die Suche nach Vorteilen, List und Täuschung, Tricks und Übertölpelung.

Was genießt also Vertrauensschutz? Was ist List, was ist Arglist? Was ist Verführung, was ist Manipulation? Wie weit geht die Selbstverantwortung?

Wie weit geht Konsumentenschutz, wie weit die Phantasie und Übertreibung der Werbung? Das sind Diskussionen, die auch politischen Modeschwankungen, das heisst einem stets wechselnden Menschenbild unterliegen.

Dieselben Fragen stellen sich in der Staatsleitung selber: Sind Listenverbindungen zulässig? Nach welchen Kriterien soll ein Abstimmungstermin festgelegt werden? Welche Vorlagen kommen gleichzeitig zur Abstimmung?

\section{Wie organisiert der Staat das Vertrauen?}

Wie wird das Grundvertrauen zwischen den Menschen hergestellt und gefördert? Kann der Staat hier etwas beitragen?

Machiavelli geht im "Fürst" davon aus, das Zusammenleben der Menschen werde wesentlich durch die Angst vor Sanktionen organisiert, denn nicht Vertrauen oder gar Liebe, sondern nur die Furcht vor dem Gesetz halte die Menschen zusammen. Ganz abgesehen davon, dass uns dies nicht als Modell eines demokratischen Staates vorschwebt, müssen wir ja auch sehen: Selbst mit uneingeschränkten polizeilichen Mitteln, selbst mit einem totalen Polizeistaat könnte ein echtes Vertrauen zwischen den Menschen erzwungen werden und garantiert werden. Der Ansatz muss daher genau umgekehrt sein: Zuerst die Freiwilligkeit, dann Anreize und erst als ultimo ratio Sanktionen.

Daher gilt auch bei uns die Freiwilligkeit als Konzept, das Vertrauen in die Bürger oder in eine Branche als Prinzip. Das Umweltgesetz oder das Energiegesetz folgen diesem Aufbau. So ist ein Torfausstiegskonzept entwickelt worden. Das Importverbot werde aber erst in 20 Jahren eingefuihrt; vorher sollen freiwillige Massnahmen greifen. Die Schweiz kennt beim freiwilligen Papier- und Karton- 
sammeln bessere Resultat als Länder, wo dies vorgeschrieben ist. Die Selbstregulierung wird jetzt auch proklamiert, um der Geldwäscherei zu bekämpfen. Die Regeln zur Bekämpfung von Schwarzgeld werden vorerst an die Branche delegiert.

Es folgen Anreize, welche die Schärfung des moralischen Bewusstseins fördern (Aufklärungskampagnen, etwa zu Toleranz im Strassenverkehr etc) und erst als letzte Massnahme Verbote und Sanktionen.

Gewiss können strafrechtliche Sanktionen die Einstellung zur Moral ändern: Fahren in angetrunkenem Zustand (FiaZ), einer Frage des Vertrauens derVerkehrsteilnehmer untereinander).

Lange galt FiaZ als Kavaliersdelikt. Noch in der parlamentarischen Beratung erfolgten von SVP Seite entsprechende Sprüche: „Ein Gläschen in Ehren, kann niemand verwehren." Doch durch die konsequente Anwendung der dann beschlossenen Strafbestimmungen erfolgte allmählich auch die gesellschaftliche Ächtung von Trunkenheit am Steuer und damit einher ging ein moralisches Umdenken, so dass jeder Strassenteilnehmer dem anderen auch das Vertrauen entgegenbringen kann, er gefährde ihn nicht durch Trunkenheit.

\section{Grundvertrauen vermag der Staat nicht zu erwirken}

Dennoch ist dies eigentlich der machiavellistische Weg zu vertrauensvollem Verhalten, nämlich die Androhung von Gefängnis und gesellschaftlicher Ächtung.

Die entscheidende Frage bleibt: Wie gelangen die Menschen zu einem Verhalten, welches gegenseitiges Vertrauen von innen heraus lebt? Wie keimt diese "Freiwilligkeit", wie sie das Gesetz postuliert, als Rückgrat unseres Verhaltens?

Das eigentliche Grundwasser des menschlichen Vertrauens, das Grund- und Gottvertrauen, das Sie soeben diskutierten, kann der Staat allein wohl kaum schaffen. Er ist angewiesen auf Religion und Kultur, die nicht nur von der rationalen Notwendigkeit des Vertrauens für das Funktionieren einer Gesellschaft ausgehen, sondern auf einer Metaebene - oder eben in den Urwogen, in den Tiefen der Seelen - das menschliche Verhalten, die Frage von Gut und Böse prägen.

Wie verhalte ich mich zu meinen Mitmenschen? Das sieht niemand im Zivil- oder Obligationenrecht nach, bevor er entsprechend fühlt oder handelt. Das verinnerlicht er, längst bevor er lesen kann. Die Quelle findet sich bei den Menschen selbst, und gespeist wird sie unter anderem aus dem ewigen Grundwasser der Kulturen, Traditionen und der Religionen. Sie haben das Gewissen von Genera- 
tionen geweckt und geschärft. Jede Gesellschaft gestaltet die Regeln des Zusammenlebens immer wieder neu. Sie greift dabei zurück auf Traditionen, Religionen. Und deswegen muss ein aufgeklärter Staat die Religions- und Kulturfreiheit gewähren, damit er selber überhaupt funktionieren kann. Der Staat - und mit ihm übrigens auch die Wirtschaft - sind also auf Religion und Kultur angewiesen, damit sie überhaupt funktionieren können. Sie müssen daher diejenigen Institutionen fördern und unterstützen, die sich dieser Aufgabe annehmen. Das sind Kirchen, Religionsgemeinschaften, das ist die Kultur. Deswegen muss der Staat Kultur und Religion Freiraum gewähren, Autonomie und Entfaltungsmöglichkeiten ermöglichen. Dazu gehören auch materielle Mittel.

Das kann bezüglich Kultur konkret etwa bedeuten, dass Sponsoring steuerlich begünstigt wird, dass die nationalen Fernsehanstalten entsprechende Aufträge erhalten. TV und Radio haben nicht nur einen Informationsauftrag, sondern auch einen Kulturauftrag zu erfüllen. Da gehört auch die Unterhaltung dazu. Die frühere Funktion von Märchen, von über Generationen hinweg weiter erzählten Geschichten und Traditionen, übernehmen heute die Massenmedien. Unterhaltung ist eine kulturelle Aufgabe und nicht nur ein samstagabendliches Geblödel. Diese Verantwortung muss der Staat in seinen Konzessionen festhalten.

Bezüglich der Religionen kann das für den Staat etwa heissen, dass Kirchen oder religiöse Gemeinden anerkannt werden, dass ihre Infrastrukturen allenfalls staatlich erleichtert oder unterstützt werden (indem zum Beispiel die Kirchensteuern durch den Staat eingezogen werden wie im Kanton Zürich). Darunter fallen dann konkrete Diskussionen darüber, ob juristische Personen Kirchensteuern bezahlen müssen oder nicht ( $\mathrm{ZH}$ : Jungfreisinnige sammeln für eine entsprechende Initiative). Die gegenseitige Abhängigkeit von Staat und Religion bedeutet für beide, dass sie sich zwar Unabhängigkeit gewähren, sich aber auch gegenseitig einem Dialog stellen müssen. Er findet heute hier statt und ich danke Ihnen dafür, dass Sie ihn organisiert haben.

— Moritz Leuenberger war von 1995 bis 2010 Mitglied des Schweizer Bundesrats und amtierte 2001 und 2006 als Bundespräsident. 